\title{
Interpretación Pragmática de los Sistemas de Creencias en Hume y Peirce
}

Pragmatic Interpretation of Belief Systems in Hume and Peirce

Dr. Alex Espinoza (alexgaston59@gmail.com) Departamento de Filosofía y Psicología, Universidad de Tarapacá (Arica, Chile)

\begin{abstract}
In philosophical literature the issue of beliefs has been identified historically with David Hume and common sense. Beliefs are dynamic systems and its resignification is constant. Charles Sanders Pierce would interpret the fixation of beliefs, as those ones which are fixed by means of art, being this a method well-tuned with science. Truths established in beliefs are always probable and dependent on the degree of utility they have. The degree of utility is complemented with comprehension, explanations have multiple causes.
\end{abstract}

Key words: belief, intelligibility, pragmatism, common sense, system.

\section{Resumen}

En la literatura filosófica el tema de las creencias se puede identificar históricamente con David Hume y el sentido común. Las creencias son sistemas dinámicos y su resignificación es constante. Charles Sanders Peirce interpretaría la fijación de las creencias en Hume, como aquella que se fija a través del arte, siendo ésta un método que se acomoda bien con el de la ciencia. Las verdades establecidas en las creencias son siempre probables y dependen del grado de utilidad que ellas presten. El grado de utilidad se complementa con la comprensión, las explicaciones son multicausales.

Palabras claves: creencia, inteligibilidad, pragmatismo, sentido común, sistema.

\section{Introducción}

Este estudio se centra en el análisis de los procesos que constituyen la lógica interna en la formación de los sistemas de creencias, siendo un tema propio de epistemología. En filosofía el tema de las creencias es un tema poco estudiado, sin embargo hemos encontrado ideas contundentes en el pensamiento de Charles Sanders Peirce y en la época moderna en David Hume. Veremos en este estudio que ambos posturas epistemológicas privilegian la opción pragmática. También se ha incluido en nuestro análisis las ideas profesadas por Niklas Luhmann sobre el concepto de sistema, ideas que confirman el pragmatismo 
como opción adecuada, y que en última instancia nos ayuda a permanecer alejados del escepticismo amenazador.

La literatura filosófica que trata el tema de las creencias nos muestra que ésta es la antítesis de la duda, y que además desempeña un rol esencial en la vida del hombre. Generalmente la creencia despierta en el hombre el sentido de seguridad y de tranquilidad; sentidos que culturalmente atesoramos. Cuando los sistemas de creencias aspiran al cambio, nos damos cuenta de la fortaleza del vínculo que nos liga a ella, así la creencia se reanima y en estos casos se resiste a ser modificada. En otras palabras, el espíritu reclama el hábito. Contrariamente a la creencia, la duda siembra la crítica, el descontento y la inseguridad, de esta manera los sistemas de creencias se sienten amenazados. La naturaleza ha dotado al ser humano de mecanismos que lo sobreprotegen ante su vulnerabilidad, es por esto que el ser humano está siempre alerta para estar fuera del alcance de las situaciones que lo conduzcan a la duda.

La afirmación anterior nos sugiere la idea y legado cartesiano: la duda en el hombre no es más que pasajera, nuestra disposición natural nos indica que debemos tender siempre hacia la creencia, existe en el hombre una fuerza que podríamos denominar natural que nos impulsa a ponernos fuera del alcance del relativismo (el racionalismo cartesiano lo tenía muy claro). Cuando cuestionamos una creencia, estamos cuestionando su fortaleza para la resolución de problemas, con ello estamos admitiendo la falibilidad de los sistemas. El fortalecimiento de la creencia radica en el mantenimiento de ella en el tiempo y al soporte a las críticas destructivas. Las creencias son sistemas que necesitan estar constantemente en vías de fortalecimiento, esto sugiere que la idea de posibilidad participa activamente en la formación de las creencias.

Los sistemas de creencias involucran cuestiones psicológicas, morales, sociológicas y físicas. Las creencias se fundamentan en estados subjetivos del hombre. Las creencias nos sirven para sostener la estabilidad emocional. En la búsqueda de la estabilidad emocional debemos tomar decisiones, luego es la voluntad con todos sus constructos axiológicos y sociales la que debe actuar. La actuación de la voluntad depende del grado de preparación que poseamos para enfrentar las eventualidades.

El fortalecimiento de las creencias se debe a mecanismos de adaptaciones que se han consolidado a través del tiempo en la vida del ser humano. Generalmente las adaptaciones dependen de cuestiones prácticas, lo verdadero se traduce en aquello que es útil y que sea benéfico para las contingencias de la vida. Así, podemos afirmar que las creencias se cimientan en una filosofía pragmática y utilitarista. Esto es válido tanto en el sentido material, como también en el sentido espiritual. El pragmatismo evoca el relativismo sugestivo, es decir, cuando nos respaldamos en el pragmatismo es porque sabemos lo que queremos. Este tipo de actitud del pragmatismo forma parte de la llamada filosofía de la acción: es estudiando la acción es que podemos considerar la vida, los sentimientos y las ideas. 


\section{Peirce y los cuatro métodos para la fijación de los sistemas de creencias}

La investigación realizada por Peirce acerca de la fijación de los sistemas de creencias es una investigación acerca de los hábitos racionales. Muchos de estos hábitos en la historia del pensamiento humano se establecen por acontecimientos históricos. Los momentos históricos nos sugieren los hábitos que son benéficos para el desarrollo de la especie humana. La elección, según Peirce, obedece a una cuestión de gusto o satisfacción, esto último lo determina lo práctico.

Según él, las creencias serían conjuntos de experiencias padecidas por el sujeto con la finalidad de controlar las eventualidades que pongan en peligro su integridad vivencial: "Sin duda, en lo fundamental, somos animales lógicos, pero no de un modo perfecto. La mayoría de nosotros, por ejemplo, somos más propensos a ser confiados y optimistas de lo que justificaría la lógica. [Más adelante afirma] La logicidad en cuestiones prácticas (si se entiende esto no en el viejo sentido, sino como consistiendo en una sabia unión de la seguridad con lo fructífero del razonar) es la cualidad más útil que puede poseer un animal, y por tanto puede derivarse de la acción de la selección natural; pero fuera de esto probablemente es más ventajoso para el animal tener la mente llena de visiones estimulantes y placenteras, al margen de su verdad; y es así por lo que la selección natural, en temas no prácticos, puede dar lugar a una tendencia falaz del pensamiento" (Peirce 2002:218).

La evolución natural ha debido ser capaz de conjugar la lógica de lo práctico con la lógica del gusto. Peirce ve que las conjugaciones han servido de modeladoras de la racionalidad, pasando por varios métodos o formas de fijación de las creencias. Peirce propone cinco métodos a través de los cuales se instauran las creencias en la vida humana con la finalidad de modelar la racionalidad, estos son: método de la tenacidad, método del autoritarismo, método metafísico, método del arte y método científico. Peirce reduce el método del autoritarismo al de la tenacidad y el método del arte al método metafísico.

\section{Método de la tenacidad}

Según Peirce un sistema de creencia instaurado por la tenacidad engendra en primera instancia una desconfianza social. El hombre no acepta que la opinión de los otros sea verdadera. "Recuerdo una vez que se me encarecía no leer un cierto periódico por miedo a que pudiese cambiar mi opinión sobre el librecambio. 'Por miedo a que pudiese quedar atrapado en sus falacias y falsedades', era la expresión. Tú no eres, decía mi amigo, un especialista en economía política. Puedes quedar por tanto fácilmente embaucado por argumentaciones falaces sobre el tema" (Peirce 2002:224).

La tenacidad, así como los métodos autoritarios, crean ambientes psicológicos impregnados de terror, en este sentido las creencias se mantienen por un largo tiempo, manteniendo, a su vez, grandes sistemas sociales. Cuando la tenacidad funda los sistemas de creencias, éstos no poseen armonía, porque el objetivo de la tenacidad es destructivo y aniquilante frente a las invasiones de sistemas adversos. 


\section{El método metafísico y del arte}

Según Peirce, el fundamento metafísico de las creencias es contrario al fundamento proporcionado por la tenacidad. La lectura metafísica enfatiza que las creencias son sistemas necesarios para justificar los sueños o los anhelos de los individuos que viven en una sociedad, estas expectativas están fundadas en la libertad: "El ejemplo más perfecto del mismo [se refiere al método metafísico] se encuentra en la historia de la filosofía metafísica. Usualmente los sistemas de este tipo no se han basado en hechos observados, al menos no a un cierto nivel relevante. Básicamente se han adoptado porque sus proposiciones fundamentales parecían agradables a la razón" (Peirce 2002:228).

Los fundamentos metafísicos de las creencias se relacionan estrechamente con la capacidad racional del hombre y por sus predilecciones, es por esto que se admite la idea de los cambios sociales y la idea del progreso, todo esto fundado en la voluntad libre de los hombres. La tenacidad no promueve la idea de los cambios en los sistemas, no se admite la presencia de hechos inesperados, éstos descompensan. La racionalidad humana como mecanismo de protección se ha dotado de apriorismos representados en el conocimiento hipotético de la realidad, de allí que nuestra vida está modelada por los experimentos mentales. Así los fundamentos metafísicos de las creencias nos protegen de las dificultades que pueden amenazar la tranquilidad humana. Cuando un sistema social está modelado por una ideología, por un constructo metafísico, podemos determinar bien las posibles amenazas.

Peirce en este paseo reflexivo de la búsqueda del fundamento ideal de las creencias, nos lleva a pensar en el arte y él considera que el arte no está ajeno a las fundamentaciones metafísicas. Ambas dependen del "gusto", de la elección libre de la voluntad. Peirce nos dice: "Cuando reaccionamos sin obstáculos, las preferencias naturales fluyen libremente en el hombre, y así se generan intercambios de sus pensamientos y consideran las cosas desde diferentes puntos de vista, esto permitió desarrollar gradualmente las creencias en armonía con las cosas naturales. Este método se asemeja a aquel que ha conducido a la madurez de las concepciones del arte" (Peirce 2002:228). Según esta cita, la naturaleza de las creencias se establece en la armonía y ésta interpretación ha estado reservada al arte, las tendencias en el arte, como también las tendencias metafísicas. Son propuestas que fluyen naturalmente desde el deseo libre y natural del ser humano. Las tendencias en el arte se eternizan justamente por su propia naturaleza: libertad y armonía.

\section{El método científico}

Las ideas anteriores expuestas nos revelan que las tendencias metafísicas y artísticas, en tanto sistemas modeladores de las creencias, son dependientes de los gustos, son sistemas que permanecen por las modas. Peirce, con el afán de encontrar un método de la fijación de las creencias que esté ajeno a los gustos y las modas, propone el método científico, y según él, es éste método el que la humanidad estuvo esperando. Estas últimas ideas evocan aquellas sostenidas por Augusto Comte respecto al positivismo. Peirce explícitamente nos dice que la induction vera de Bacon es lo que estábamos esperando (1). De la misma manera que Bacon y Comte, Peirce nos dice que el método científico es el que debe sacar las creencias de la arbitrariedad y de la accidentalidad. Este método, dice Peirce, debe estar ajeno a la historia 
humana y por tal situación es común para todos seres pensantes: "Y aun cuando tales afecciones son necesariamente tan diversas como lo son las condiciones individuales, con todo el método ha de ser tal que la conclusión última de cada una sea la misma. Tal es el método de la ciencia" (Pierce 2002:230).

Estas ideas constituyen el pilar del realismo científico y Mario Bunge es considerado como uno de los mejores exponentes. Él sostiene que la ciencia es la encargada de explicarnos la realidad y que esto es posible gracias a los cuerpos teóricos bien elaborados, aun cuando ellos no sean más que modelos representativos. Los modelos teóricos pueden variar en el tiempo si la realidad así lo exige. Él propone una concepción hipotética de la verdad o una concepción incompleta de las verdades científicas. Esto último no quiere decir que el sentido de la objetividad se pierda y que la ciencia corra el riesgo de caer en una especie de subjetivismo relativista o en el anarquismo, sino que la ciencia posee sus propios mecanismos experimentales que permiten eludir estos problemas. De esta manera las verdades científicas son las responsables de la modelación genuina de las creencias, las verdades que ponemos en duda se corrigen a través de la aplicación del mismo método.

Peirce en su artículo dedicado a la Fijación de las Creencias concluye en una tesis auspiciada por su pragmatismo: él afirma que los sistemas de creencias fundados ya sea en la tenacidad, en el arte o en la metafísica, han sido sistemas oportunos para los momentos históricos que los vieron nacer, y cada uno de ellos no son producto de un plan deliberado de la racionalidad humana, como sería el caso de los sistemas de creencias fundado en la ciencia.

Sería interesante analizar los sistemas de creencias desde una perspectiva diferente. Para este propósito recurriremos a las ideas de David Hume, cuya filosofía está dedicada, en gran parte, al sentido común. De estos estudios emergen los problemas de la inducción, es decir, ¿cómo el sentido común establece generalidades? o ¿cómo el sentido común predice hechos a futuro? Este pensador sostendrá que el tema de las creencias es fundamental para responder parcialmente a la problemática de la inducción y con ello este tema es propio de la subsistencia de la especie humana.

\section{Hume: la participación de la psicología, de la biología y de la sociología en la fijación de las creencias}

David Hume en su gran obra A Treatise of Human Nature, publicada en 1739, estudia los principios que gobiernan el entendimiento humano. En esta obra analiza los sistemas de creencias considerándolos como esenciales para la vida humana. Así, él llega a la clara convicción que las creencias son sistemas que gobiernan la vida humana, pero al mismo tiempo las creencias son sistemas sujetos a eventualidades determinadas por las circunstancias históricas del sujeto. La predicción de las acciones humanas son imposibles de determinar con exactitud. Existiría una especie de determinismo histórico débil, estas ideas validan en algún sentido el problema de la inducción.

Para Hume el control del determinismo histórico se fundaría en el formalismo: el cálculo de probabilidades: "Existe una cierta probabilidad, que nace de una superioridad de las chances por un lado; en proporción al aumento de esta superioridad y de esta predominancia sobre las chances contrarias, la probabilidad aumenta y ella engendra un grado aún más alto de creencia o de aprobación, así descubrimos 
la superioridad" (Hume 1999:131) (2). La probabilidad es la manera formal de determinar el grado de credibilidad de las creencias. Ella genera en el individuo mezclas de estados psicológicos y, a partir de esto, logramos restablecer la propensión y la seguridad para tomar decisiones. Para Hume, las creencias (belief) comprenden mecanismos psicológicos que aportan la seguridad al sentido común. El hombre puede involucrarse con la realidad pensando que puede utilizar ideas seguras que lo conducirán exitosamente hacia las metas propuestas: en Hume encontramos el germen de una concepción pragmática en las actividades humanas.

Se ha hecho notar que en la filosofía humeana los sistemas de creencias son esenciales para el conocimiento del sentido común. Es por esta razón que Hume en la época moderna se considera como uno de los mejores exponentes en el estudio de la cotidianidad (3). Sin embargo, Hume está consciente que el entendimiento humano está modelado también por el conocimiento científico, y que este tipo de conocimiento se rige por principios lógicos que han constituido la filosofía racional, principios como: la causalidad, la identidad y todas aquellas ideas que provengan de una actitud racionalista. Hume prefiere reducir el conocimiento del sentido común a procesos fundados en la imaginación; la imaginación escapa al determinismo causal, a la identidad propia de los objetos que adornan el mundo. La imaginación sería la única facultad capaz de conocer la realidad en el continuo (4). Las creencias son sistemas dinámicos que admiten el cambio constante, el remodelamiento, las creencias viven apegadas a la realidad, determinadas por las coordenadas de tiempo y de espacio. La imaginación, según Hume, es la única facultad humana capaz de acompañar el estado dinámico de la realidad, ella sugiere al sentido común lo que es conveniente hacer, aquí lo que prevalece es la acción supeditada al éxito. El éxito de nuestras acciones depende del grado de exactitud de las creencias, a este respecto es imprescindible el grado de confiabilidad de nuestra acción, de allí el llamado de Hume al escepticismo mitigado.

Como resultado de nuestros análisis sobre el tema de la fijación de las creencias, podemos afirmar que tanto Hume como Peirce consideran que las creencias sostenidas por el sentido común se acomodan a la interpretación del arte o a la interpretación metafísica, vale decir, para vivir en armonía en la cotidianeidad es necesario aceptar los cambios de manera sensata. La sensatez de los hombres radica en el grado de satisfacción que se produzcan en ellos, como plantearía el pragmatismo de Peirce: "es una cuestión de gusto". La armonía en el arte es la belleza, la armonía en la vida humana es la paz concebida en la acción. Así, para Hume la creencia tiene su origen en factores naturales biológicos y psicológicos, vale decir, la paz para la acción humana es una cuestión esencial para la sobrevivencia de la especie humana. Los factores sociológicos e históricos que moldean las creencias son posteriores: "La naturaleza puede ciertamente producir todo aquello que nace del hábito; aún mejor, el hábito no es otra cosa que uno de los principios de la naturaleza y ella obtiene toda su fuerza de este origen" (Hume 1985:229) (5).

Otra forma de comparar las ideas de Hume respecto a las de Peirce, consiste en la clara convicción de Peirce de pensar que la ciencia es la única forma válida y universal de fijar las creencias. Hume pensará que ésta es una manera legítima de brindar seguridad en el conocimiento, de allí su célebre conclusión de su obra An enquiry concerning human understanding: "El caso es el mismo para todos los razonamientos pertenecientes a la silogística y que podemos encontrar en todas las ramas del saber, como es el caso de 
las ciencias de la cantidad y del número; podemos afirmar, entonces, con toda certeza, yo pienso, que la cantidad y número son los únicos objetos propios del conocimiento y de la demostración" (Hume 1999:129) (6). Sin embargo, Hume también piensa que las creencias provenientes del sentido común constituirán otra forma de conocimiento, que Hume la legítima como válida o más bien como necesaria para nuestro sistema de vida.

\section{¿Las creencias se fundamentan en la experiencia sensible o en la inteligibilidad?}

Para el sentido común, el realismo ingenuo ha constituido una de las justificaciones mejor aceptadas para dar cuenta de la fijación de las creencias. La tesis principal que sostiene el realismo ingenuo se define por una especie de determinismo sensitivo, vale decir, el sujeto se encuentra determinado por las experiencias sensibles surgidas de las circunstancias. Estas ideas proporcionadas por el realismo ingenuo se complementarían bien con las conclusiones pragmáticas aludidas por Hume y Peirce, y que ya hemos analizado: "las creencias se fijan en última instancia por gusto y porque es benéfica para la subsistencia". Pero cuando el sentido común fija las creencias: ¿prima en él la verificación?, es decir, para creer ¿se necesita que el acontecimiento se muestre en un tiempo y un espacio determinado? o ¿basta que se produzca en el sujeto un sentimiento especial? En otras palabras, para creer que el sol saldrá mañana, basta haber constatado que el sol hasta el día de hoy ha salido o ¿estamos seguros que saldrá mañana porque se despierta en mí un sentimiento especial? La consideración del sentimiento, vale decir, de una condición psicológica, es una característica propia de la inteligibilidad, ésta requiere por una parte de la suscitación del sentimiento, y por otra parte requiere la manifestación material del acontecimiento. En la inteligibilidad no hay una separación abrupta entre la materialidad del mundo y el espíritu, la materialidad se reconfigura en la observación directa o indirecta y así nace una sensación propia de un estado espiritual. En la inteligibilidad se cruzan aspectos racionales y emocionales. Cuando algo se entiende o intelige, es toda la persona que se ve comprometida.

En los sistemas de creencias que se establecen ya sea por la vía científica, ya sea por la vía del arte o de la metafísica, predominan la inteligibilidad porque, como hemos afirmado, cada una de ellos se fundamentan en el gusto, y el gusto es una cuestión de sensación. Así, se despierta una emoción que nos conducirá hacia la tranquilidad. No olvidemos que la sabiduría griega se define por la conquista de la verdad y ésta depende de la inteligibilidad, esta despierta la admiración, la armonía, la felicidad.

Si bien es cierto que los sistemas de creencia (fundados en la metafísica, el arte o la ciencia) establecidos por el sentido común certifican sus verdades por los hechos observados y acontecidos, no es menos cierto que se busca el entendimiento profundo, se busca que se suscite un sentimiento particular para la aprobación, esto último nos define como seres humanos. Si tanto Hume como Peirce privilegian la actitud pragmática ante el problema de la fijación de las creencias, ellos deben consecuentemente aceptar la idea que los sistemas de creencias se establecen por cuestiones prácticas y emocionales. Lo "práctico" en sí encierra cuestiones de índole emocionales, en última instancia de comodidad, la inteligibilidad está presente con todo su esplendor en el momento en que se fijan los sistemas de creencias. 


\section{Hume y Peirce: la teoría de sistemas y las creencias}

El modelo de racionalidad que gobernó la edad moderna fue aquel que estaba representado por Isaac Newton en su gran obra De Philosophiae Naturalis Principia Mathematica. Sabemos bien que este ideal modelador de conocimiento traspasó las fronteras de cada disciplina científica pertenecientes a las ciencias naturales, e influyó en su desarrollo, siendo el unicausalismo su gran aliado. Por el contrario, el positivismo en las ciencias sociales nunca se ha sentido cómodo, de allí que se reclame exactitud en los sistemas que gobiernan nuestras experiencias cotidianas, pero el sentido común, objeto de estudio esencial de las ciencias sociales, es dependiente de las creencias y las creencias son sistemas que se fijan o establecen multicausalmente. Una creencia se fija cuando ya han pasado una serie de eventos, y cuya sumatoria indica que lo establecido es benéfico para el sistema, nos indica que el sistema está estable. Así, la epistemología ha ido delineando la teoría de sistemas como alternativa explicativa de los fenómenos sociales.

Pensar las creencias como sistema significa que no existe una causa privilegiada, sino más bien, un sistema es un todo y reacciona como un todo, si se altera una parte de él, entonces se modifica el todo. Uno de los grandes sistémicos, como es el caso de Niklas Luhmann, piensa que las explicaciones relacionan conjuntos de causas con conjuntos de efectos: "El futuro ya no está obstruido por fines previamente dados y verdaderos sino están abiertos hasta la infinitud, contiene más posibilidades de las que pueden ser actualizadas y debe, pues, ser fijado por medio de planes" (Luhmann 1983:23). Respecto a esta cita, Luhmann diría que las creencias se constituyen en forma de planes, se establecen porque existe una teleología implícita, hay metas y fines claros que ordenan sus prioridades. El funcionamiento de las creencias se determina, según Luhmann, porque existen códigos axiológicos estables, porque las escalas valorativas son las que permiten determinar la calidad de los sistemas de creencias. El pensamiento de Luhmann es claro: la estabilidad de un sistema de creencias se define no como un sistema estático, sino como un sistema altamente dinámico. Un sistema rígido exige, afirma Luhmann, la petrificación de los códigos valóricos. El sistema de creencia, en este sentido, no aceptaría resignificaciones de las creencias, como por ejemplo, los sistemas de creencias establecidos por la vía de la tenacidad propuesto anteriormente por Peirce, no hay intercambio de energía, de información, así la constitución racional del sistema será problemática.

Nuestros pensadores como Hume y Peirce ¿estarían de acuerdo en aceptar la interpretación sistémica y dinámica de las creencias? Tanto Hume como Peirce están de acuerdo en interpretar los sistemas de creencias como sistemas que fijan sus verdades en función de los beneficios que traen para su subsistencia, luego, esta idea despierta el dinamismo que debe existir en el seno interno de un sistema de creencia. Como afirma Luhmann, debe haber un intercambio constante de energía entre los medios y los fines, y nunca se debe perder de vista el contexto en cual este dinamismo se desarrolla. Analógicamente, los sistemas biológicos evolucionan porque están apegados a los cambios de los nichos ecológicos, en forma similar, los sistemas de creencias evolucionan porque están apegados a los vaivenes de la historia humana. 
Desde esta perspectiva podemos afirmar que la interpretación sistémica es válida para conocer y determinar los procesos que ocurren en los sistemas de creencias. Las creencias para mantenerse en el tiempo necesitan de una serie de acontecimientos entrelazados que son difíciles de separar. Esto último cuestiona los enmarques positivistas clásicos, las explicaciones unicausalistas o la separabilidad de los sistemas, son ideales difíciles de cumplir en ciencias sociales.

\section{Conclusión}

En resumen, podemos concluir que las relaciones filosóficas entre Hume y Peirce testimonian un fundamento pragmático de los sistemas de creencias, siendo estos, a la vez, el fundamento último de la vida.

En la filosofía de David Hume, gracias a los sistemas de creencias, el conocimiento del sentido común frena su caída en el escepticismo radical. Comparativamente en la filosofía de Charles Sanders Peirce las creencias establecidas por la vía metafísica y por la vía del arte son complementarias a las creencias científicas. Hume y Peirce quieren estar alejados del escepticismo, esto es el principio fundamental de las creencias.

Tanto Hume como Peirce interpretan la fijación de las creencias como momentos propios de la inteligibilidad. Queremos vivir en tranquilidad, este es un logro cuando la comprensión profunda se establece, para esto último debemos tener satisfechas por una parte las necesidades que exige nuestra naturaleza evolutiva, y por otra parte, debemos sentirnos feliz en ella, las creencias de origen natural como las de origen cultural son esenciales.

Las creencias se explican como sistemas, las variables que se entrecruzan son múltiples, pero en que cada creencia hay fines o metas claras que están implícitamente presentes en cada acción, la teleología implícita está alerta a las modificaciones del entorno, ellas están en estado constante de resignificación. Estas ideas de Luhmann se avienen con el escepticismo mitigado de Hume y con el pragmatismo de Peirce (7).

\section{Notas}

(1) La cita textual en donde Peirce expone la tesis baconiana dice: "Y así, a partir de este método, que se ha llamado el método a priori, [se refiere al método metafísico] llegamos, en frase de Lord Bacon, a la verdadera inducción" (2002:229).

(2) De ahora en adelante cada cita es traducción mía. Cita textual en ingles: "There is certainly a probability, which arises from a superiority of chances on any side; and according as this superiority increases, and surpasses the opposite chances, the probability receives a proportionable increase, and begets still a higher degree of belief or assent to that side, in which we discover the superiority" (Hume 1999:131).

(3) En la misma dirección del análisis de Hume, Thomas Reid, filósofo de la escuela escocesa del sentido común y contemporáneo de Hume, piensa que el sentido común determina la verdad de los sistemas y éste a la vez determina la intencionalidad y la interpretación de los signos: "El sujeto es el intérprete de 
este signo, pero sólo puede interpretarlo en la medida que posea concepciones previas del objeto representado. Entonces el signo no consigue explicar la intencionalidad, porque lo presupone antes de la relación cognoscitiva sujeto-objeto-representación" (Espinoza y Soto 2011:156).

(4) Contrario de lo que podría pensarse, la ciencia sobre todo hoy día está obligada a construir sistemas fundados en la imaginación para explicar y entender el orden de las cosas en el mundo de lo infinitamente pequeño. La imaginación permite llenar los vacíos que la racionalidad ha ido dejando, la imaginación está al servicio de la racionalidad.

(5) Nature may certainly produce whatever can arise from habit: Nay, habit is nothing but one of the principles of nature, and derives all its force from that origin (Hume 1985:229).

(6) It is same case with all those pretended syllogistical reasonings, which may be found in every other branch of learning, except the sciences of quantity and number; and these may safely, I think, be pronounced the only proper objects of knowledge and demonstration" (Hume 1999:129).

(7) El artículo forma parte del proyecto de investigación 3733-12 de la Universidad de Tarapacá.

\section{Bibliografía}

Espinoza, A y Soto, M. 2011. La participación de los entes inobservables en el problema cuerpo-mente. Armonía entre el intelecto y la naturaleza. Revista Alpha 33: 147-158.

http://dx.doi.org/10.4067/S0718-22012011000200011

Hume, D. 1999. An enquiry concerning human understanding. Oxford: Oxford University Press.

Hume, D. 1985. Treatise of human nature. Oxford: Penguin Books.

Luhmann, N. 1983. Fin y racionalidad de los sistemas. Madrid: Nacional.

Peirce, C. 2002. Pragmatisme et pragmaticisme. Paris: Du Cerf.

Recibido el 4 Ene 2014

Aceptado el 18 Jun 2014 